\title{
Clinical and microbiological features of Actinotignum bacteremia: a retrospective observational study of 57 cases
}

\author{
H. Pedersen ${ }^{1}$ E. Senneby ${ }^{1,2} \cdot$ M. Rasmussen ${ }^{1}$
}

Received: 24 October 2016 / Accepted: 30 November 2016/Published online: 12 December 2016

(C) The Author(s) 2016. This article is published with open access at Springerlink.com

\begin{abstract}
The purpose of this study was to investigate the incidence, clinical presentation, and prognosis of Actinotignum bacteremia in southern Sweden. Actinotignum isolates in blood cultures were identified retrospectively between 1st January 2012 and 31st March 2016 through searches in the clinical microbiology laboratory database. The population covered by this laboratory is approximately 1.3 million. Matrix-assisted laser desorption/ionization timeof-flight mass spectrometry (MALDI-TOF MS) was used for species determination. Etests were used for minimum inhibitory concentration (MIC) determination. The patients' medical charts were reviewed. Fifty-eight episodes in fifty-seven patients with Actinotignum bacteremia were identified (A. schaalii $=53$, A. sanguinis $=1$, A. urinale $=2$, and Actinotignum species $=3$ ), which corresponds to an incidence of 11 cases per million inhabitants. Fifty-one percent of the isolates were in pure culture. The MICs were low for $\beta$-lactam antibiotics, whereas high MICs were recorded for ciprofloxacin and trimethoprim. Patients had a median age of 82 years, $72 \%$ were male, and a majority had underlying urological conditions. Thirty-six of the patients were diagnosed with a focus from the urinary tract. Thirty-one patients developed severe sepsis and nine patients died during the hospital stay. Our study is the largest of Actinotignum bacteremia and demonstrates that it is a condition with a significant fatality that affects elderly persons with underlying conditions. $\beta$-Lactams represent a rational treatment option.
\end{abstract}

M. Rasmussen

Magnus.Rasmussen@med.lu.se

1 Department of Clinical Sciences, Division of Infection Medicine, Lund University, BMC, B14, Tornavägen 10, 22184 Lund, Sweden

2 Clinical Microbiology, Region Skåne, Lund, Sweden

\section{Introduction}

The genus Actinobaculum was first described in 1997, when it was separated from Actinomyces $[1,2]$. Several species belonging to Actinobaculum were reclassified in 2015 and they were proposed to constitute a novel genus, Actinotignum [2]. This genus now comprises three species: Actinotignum schaalii, Actinotignum sanguinis, and Actinotignum urinale [2]. These bacteria are small facultative anaerobic Grampositive rods that are non-motile and catalase-negative [1]. They grow slowly and preferably under anaerobic or $\mathrm{CO}_{2}$ conditions, and are easily overgrown by other bacteria [3]. Actinotignum species are probably part of the normal urogenital flora [4] and have been associated with urinary tract infections $[5,6]$. They are, however, most likely under-diagnosed in urine cultures, since most clinical microbiological laboratories routinely only use aerobic growth conditions. Matrixassisted laser desorption/ionization time-of-flight mass spectrometry (MALDI-TOF MS) appears to be a useful method for correct species determination of these bacteria [3].

Actinotignum schaalii has been associated with urinary tract infections [6, 7] but can also cause invasive infections, such as Fournier's gangrene, urinary bladder necrosis, bacteremia, and endocarditis [8-11]. The characteristics of infections with $A$. schaalii have been described in two recent reviews $[5,12]$. However, only a few studies have addressed the clinical presentation of Actinotignum bacteremia. In a study from Denmark, 98 patients with A. schaalii bacteremia were identified between 2011 and 2014, but the clinical presentation was only described for ten patients [8]. These had a median age of 79 years, $70 \%$ were male, and $50 \%$ had predisposing urogenital conditions. In a Swedish study, 17 cases of A. schaalii bacteremia were identified retrospectively and patients were shown to typically be older males with underlying urogenital risk factors [13]. Similar results have been 
indicated in a case series from Spain [14], as well as in reports of single cases and smaller case-series [7, 15-20]. Given the low number of cases reported, the prognosis of Actinotignum bacteremia is not known.

In published cases, the preferred treatment of $A$. schaalii bacteremia has been with $\beta$-lactams to which the bacterium has low minimum inhibitory concentration (MIC) values. However, since bacteremia typically has a urinary tract focus, there is a risk that empiric treatment will be given with antibiotics such as trimethoprim-sulfamethoxazole or ciprofloxacin, to which the bacterium is resistant in vitro $[16,19,21]$. The duration of the treatment is still uncertain but there have been failures after 1 week of ampicillin treatment $[6,19]$, which indicates that longer treatment periods may be needed. In this retrospective study, we present the incidence, clinical presentation, and prognosis of Actinotignum bacteremia in southern Sweden.

\section{Materials and methods}

\section{Setting}

Patients with any Actinotignum species in blood cultures between 1st January 2012 and 31st March 2016 were identified through searches in the database belonging to the clinical microbiology laboratories in Malmö and Lund, Sweden. This database contains clinical samples from all ten hospitals within Region Skåne, with 1,303,627 inhabitants in 2015 [22]. The laboratories used blood culture systems and MALDI-TOF MS, as previously described [23]. We performed species determination with $16 \mathrm{~S}$ rRNA gene sequencing with fD1 mod and P911 primers, as previously described [24], on all isolates where both the initial and renewed MALDI-TOF MS analysis gave a score below 2.0 .

\section{Clinical presentation}

The medical charts were studied and the following variables were extracted: age, underlying diseases, urogenital risk factors, symptoms, vital signs, laboratory values, antibiotic treatment, diagnosis, outcome, and other microbiological findings. Urogenital risk factors were defined as one or more of catheterization, prostatic hyperplasia, prostatic cancer, bladder cancer, hydronephrosis, renal failure, urethral stricture, or kidney stones. Severe sepsis was defined as one or more of hypotension with a systolic blood pressure below $90 \mathrm{mmHg}$, hypoperfusion with plasma lactate of $>3.3 \mathrm{mM}$, or organ dysfunction. Organ dysfunction, in turn, was defined as an increase in plasma creatinine by $>44 \mu \mathrm{mol} / 1$, saturation $<86 \%$ at admission ( $<78 \%$ if the focus of infection was the lungs), platelets $<100 \times 10^{9} / 1$ or $\mathrm{PK} / \mathrm{INR}>1.5$, an acute confusion and/or bilirubin $>45 \mu \mathrm{mol} / 1$ [25]. A need for ventilation support, inotropic support, or continuous hemofiltration was also regarded as proof of organ dysfunction.

\section{Antibiotic susceptibility}

Isolates were cultured for $48 \mathrm{~h}$ under anaerobic conditions (Whitley A35 Anaerobic Workstation) on blood agar plates [produced by the Clinical Microbiology Laboratory, Lund, Sweden, using Blood Agar Base LAB028 (LabM, Lancashire, UK), with the addition of $5 \%$ horse blood]. The MIC was determined using the Etest (bioMérieux, Marcyl'Etoile, France) on Mueller-Hinton fastidious agar plates according to the instructions give by the manufacturer. The MIC was recorded after 24 or $48 \mathrm{~h}$ of incubation anaerobically.

\section{Statistical methods}

Continuous variables were compared using the MannWhitney $U$-test and categorical variables using the Fisher's exact test in Prism 7 for Mac OS X.

\section{Results}

\section{Microbiology}

A total of 58 episodes of Actinotignum bacteremia were identified, corresponding to an incidence of 11 cases per million inhabitants per year. Fourteen of the isolates had an MALDITOF MS score below 2.0 and were subjected to sequencing of the $16 \mathrm{~S}$ rRNA gene for species determination. Eleven isolates were identified as $A$. schaalii, whereas three of the isolates could not be securely identified to the species level and were, thus, designated as Actinotignum species. Actinotignum schaalii was isolated from 53 patients, A. sanguinis was isolated from one patient, and A. urinale was isolated from two patients (once with $A$. schaalii and once with Peptoniphilus harei). One patient had a recurrent $A$. schaalii infection, with six months between the episodes, and only the first episode is reported here. In total, Actinotignum was the only finding in blood in 29 cases. In the other cases, additional bacterial species were isolated from the blood. The most common findings were Aerococcus species $(n=9)$, Peptoniphilus species $(n=4)$, Escherichia coli $(n=3)$, Enterococcus faecalis $(n=3)$, and Proteus mirabilis $(n=3)$. In four samples, more than two bacterial species of bacteria were isolated. The most common bacteria found in urine were $E$. coli $(n=14)$, E. faecalis $(n=5)$, and Aerococcus species $(n=3)$. Only one urine sample grew $A$. schaalii.

Of the 54 isolates available for antimicrobial susceptibility testing, all showed low MIC values to $\beta$-lactams and vancomycin, whereas the MIC values varied for erythromycin, clindamycin, and gentamicin. High MICs were recorded for 
trimethoprim and ciprofloxacin. The results are summarized in Table 1. European Committee on Antimicrobial Susceptibility Testing (EUCAST) breakpoints for Grampositive anaerobes were used for classification of sensitivity [26].

\section{Clinical presentation}

The median age of the patients was 82 years and $72 \%$ were men. Only one of the patients had no underlying conditions. A majority of the patients $(61 \%)$ had one or more underlying urogenital conditions, the most common being prostatic disorders $(n=16)$ and urinary catheter $(n=14)$. The patient characteristics are summarized in Table 2. The most common symptom at presentation was fever $(61 \%)$. Nine of the patients presented with symptoms from the urinary tract, such as hematuria or strong smelling urine, and nine presented with unspecified abdominal pains. In eight subjects, the positive blood culture was drawn after more than $48 \mathrm{~h}$ of hospitalization and, thus, represent nosocomial infections.

\section{Course and outcome}

Thirty-one patients developed severe sepsis, and in 39\% of these cases, more than one organ failure was recorded. Thirteen of the patients with severe sepsis had Actinotignum as the single organism isolated from blood, whereas 18 of these patients had polymicrobial bacteremia. Renal failure was the most common organ failure $(n=17)$, followed by hypoperfusion $(n=10)$ and respiratory failure $(n=8)$. Five patients were treated in the intensive care unit with noninvasive ventilation or vasopressor support. Thirty-six patients were diagnosed with a focus in the urinary tract and 11 with pneumonia, though chest radiograms were not conclusive for pneumonia in any of these cases. Eight patients were subjected to echocardiography, which did not reveal signs of endocarditis. Fifty-three patients received adequate antibiotic treatment initially, of which 43 were treated with $\beta$-lactams. The most common follow-up treatment was per oral amoxicillin $(n=19)$. A few patients received ciprofloxacin as follow-up. The median time of the total antibiotic treatment was 14 days. The median time of hospitalization was 10 days for survivors. Nine of the patients died during hospitalization. The features of deceased patients and survivors are given in Table 3 .

\section{Discussion}

The number of reported cases of Actinobaculum/Actinotignum bacteremia has been very low, possibly due to the relatively recent identification of the species and missed identification in the microbiological laboratories. With MALDI-TOF MS as the primary species identification method and an increasing number of older persons with urinary tract morbidity, Actinotignum infections will likely be more frequently encountered. We report an incidence of Actinotignum bacteremia of 11 cases per million inhabitants per year, which is higher than what is suggested by a recent Danish study [8]. Our results confirm previous studies showing that patients affected by the condition are typically older males with underlying urinary tract conditions [8, 13, 14]. From our case series, which is the largest one to date, the prognosis of Actinotignum bacteremia can be assessed. We report a relatively high mortality (16\%), which is likely related to the advanced age and co-morbidity of the patients. Some of the deceased patients had polymicrobial bacteremia and the exact contribution of A. schaalii to the outcome is difficult to determine.

Actinotignum schaalii is the most commonly isolated Actinotignum species both in our study and in previous reports $[6-8,13]$. We identified one case of A. sanguinis and two cases of $A$. urinale bacteremia. However, MALDI-TOF MS

Table 1 Minimum inhibitory concentration (MIC) values for the 54 Actinotignum isolates

\begin{tabular}{|c|c|c|c|c|c|c|c|c|c|c|c|c|c|c|c|c|c|c|}
\hline \multirow[t]{2}{*}{ Antibiotics } & \multicolumn{18}{|c|}{ MIC values } \\
\hline & 0.002 & 0.004 & 0.008 & 0.016 & 0.032 & 0.064 & 0.125 & 0.25 & 0.5 & 1 & 2 & 4 & 8 & 16 & 32 & 64 & 128 & $>256$ \\
\hline Bensylpenicillin & & 2 & 9 & 26 & 14 & 3 & & & & & & & & & & & & \\
\hline Ampicillin & & & & 4 & 2 & 2 & 6 & 15 & 16 & 5 & 4 & & & & & & & \\
\hline Cefotaxime & & & 12 & 15 & 16 & 4 & 6 & 1 & & & & & & & & & & \\
\hline Imipenem & 1 & 3 & 16 & 23 & 10 & & 1 & & & & & & & & & & & \\
\hline Vancomycin & & & & & & 1 & 19 & 31 & 2 & & 1 & & & & & & & \\
\hline Gentamicin & & & & & & & & & 2 & 15 & 32 & 1 & & 3 & & 1 & & \\
\hline Ciprofloxacin & & & & & & & & & 1 & 1 & 4 & 4 & 3 & 3 & 38 & & & \\
\hline Trimethoprim & & & & & & & & & & & & & & & 54 & & & \\
\hline Clindamycin & & & & 19 & 16 & 10 & 1 & 1 & 1 & & & & & & & & & 6 \\
\hline Erythromycin & & & & 20 & 17 & 10 & & & 1 & & & & & & & & & 6 \\
\hline
\end{tabular}


Table 2 Clinical features of Actinotignum bacteremia

\begin{tabular}{|c|c|}
\hline \multicolumn{2}{|l|}{ Demographics } \\
\hline Median age (years) & 82 (range 48-98) \\
\hline Male sex & $41(72 \%)$ \\
\hline \multicolumn{2}{|l|}{ Microbiology } \\
\hline Pure culture & $29(51 \%)$ \\
\hline Positive urine cultures & $26(46 \%)$ \\
\hline \multicolumn{2}{|l|}{ Underlying conditions } \\
\hline Urogenital conditions $^{\mathrm{a}}$ & $35(61 \%)$ \\
\hline Cardiovascular disease $\mathrm{e}^{\mathrm{b}}$ & $39(68 \%)$ \\
\hline Dementia & $8(14 \%)$ \\
\hline Diabetes mellitus & $11(19 \%)$ \\
\hline \multicolumn{2}{|l|}{ Symptoms and signs } \\
\hline Fever & $35(61 \%)$ \\
\hline Urinary tract symptoms & $9(16 \%)$ \\
\hline Respiratory symptoms & $17(30 \%)$ \\
\hline Severe sepsis & $31(54 \%)$ \\
\hline Median $\mathrm{CRP}^{\mathrm{c}}$ at hospitalization & $59 \mathrm{mg} / \mathrm{L}$ (range $2-420$ ) \\
\hline Median $\mathrm{WBC}^{\mathrm{d}}$ at hospitalization & $\begin{array}{l}14 \times 10^{9} / \mathrm{L} \text { (range } \\
\quad 5.8-50)\end{array}$ \\
\hline \multicolumn{2}{|l|}{ Focus of infection } \\
\hline Urinary tract & $36(63 \%)$ \\
\hline Unknown & $19(33 \%)$ \\
\hline Other & $2(4 \%)$ \\
\hline \multicolumn{2}{|l|}{ Management and outcome } \\
\hline $\begin{array}{l}\text { Initial antibiotic effective against } \\
\text { Actinotignum }\end{array}$ & $53(93 \%)$ \\
\hline Median in-hospital time (days) & 10 (range $2-25$ ) \\
\hline Echocardiogram & $8(14 \%)$ \\
\hline $\mathrm{ICU}^{\mathrm{e}}$ & $5(9 \%)$ \\
\hline Deceased during hospitalization & $9(16 \%)$ \\
\hline
\end{tabular}

${ }^{\mathrm{a}}$ Underlying conditions defined as one or more of catheterization, prostatic hyperplasia, prostatic cancer, bladder cancer, hydronephrosis, renal failure, urethral stricture, lower urinary tract symptoms (LUTS), or kidney stones

${ }^{\mathrm{b}}$ Underlying conditions defined as atrial fibrillation, pacemaker, ischemic heart disease, myocardial infarction, or stroke

${ }^{\mathrm{c}} \mathrm{C}$-reactive protein

${ }^{\mathrm{d}}$ White blood cells

${ }^{\mathrm{e}}$ Intensive care unit

was unable to reliably identify all isolates to the species level. Sequencing of the 16S rRNA gene proved to have a limited ability to separate $A$. schaalii from $A$. sanguinis and, therefore, some isolates could not be identified to the species level.

There are many similarities between $A$. schaalii and Aerococcus urinae [27]. Both species probably colonize the urinary tract and they have been difficult to correctly identify in the past. They cause invasive infections mostly in older males with underlying urogenital conditions, and are resistant to antibiotics commonly used to treat urinary infections. In fact, Actinotignum species and Aerococcus species have been isolated together from blood previously $[18,23]$ and, in our
Table 3 Features of survivors and the deceased

\begin{tabular}{llll}
\hline & Survivors & Deceased & $\begin{array}{l}p \text { - } \\
\text { Value }\end{array}$ \\
\hline Median age & & & \\
Male sex & 81 years & 86 years & 0.03 \\
Pure culture & $33 / 48(69 \%)$ & $8 / 9(89 \%)$ & 0.4 \\
Severe sepsis & $26 / 48(54 \%)$ & $2 / 9(22 \%)$ & 0.1 \\
Median CRP ${ }^{\text {a }}$ at hospitalization & 58 & 59 & 0.3 \\
Median WBC ${ }^{b}$ at hospitalization & 13 & 16 & 0.3 \\
Underlying urogenital conditions & $29 / 48(60 \%)$ & $5 / 9(56 \%)$ & 1 \\
Urinary tract focus of infection & $32 / 48(67 \%)$ & $3 / 9(33 \%)$ & 0.1 \\
Nosocomial infection & $5 / 48(10 \%)$ & $2 / 9(22 \%)$ & 0.3 \\
Adequate initial antibiotic treatment & $44 / 48$ & $9 / 9$ & 1 \\
\hline${ }^{a}$ C-reactive protein & & & \\
${ }^{b}$ White blood cells & & &
\end{tabular}

material, Aerococcus was the most common finding in polymicrobial Actinotignum bacteremia. Our previous description of Aerococcus bacteremia and the present description of Actinotignum bacteremia, however show some differences [23, 28, 29]. The mortality is higher in Actinotignum bacteremia as compared to Aerococcus bacteremia (16 vs. $8 \%)[18,23]$. This could either be due to specific bacterial virulence factors expressed by Actinotignum or also be due to host factors such as more advanced underlying conditions. Another difference is that Actinotignum species rarely seem to cause infective endocarditis as opposed to Aerococcus species, which cause endocarditis in a significant proportion of cases of bacteremia [23, 30, 31]. In our series, no case of endocarditis was found and only a single case of endocarditis caused by Actinotignum has been reported previously [9].

Only in one of the patients was A. schaalii isolated from urine. This is most likely due to routine practices in the clinical microbiology laboratory, which are unfavorable for the detection of Actinotignum species. Our findings, however, suggest that the urinary tract was the focus of the bacteremia in a majority of the cases. Many patients had underlying urological conditions and presented with signs and symptoms from the urinary tract. The patients with polymicrobial bacteremia had other typical uropathogens in their blood and urine cultures, such as Aerococcus species, E. coli, and E. faecalis, which also suggest a urinary tract focus. The patients who received a clinical diagnosis of pneumonia did not fulfill criteria necessary for that diagnosis and most of them likely had a urinary tract focus of infection.

There are no clinical break points established for Actinotignum species. However, from our results on antimicrobial susceptibility testing, $\beta$-lactams and vancomycin seem like feasible treatment options. Trimethoprim and ciprofloxacin display high MIC values and should be avoided. Previous studies have reported relapse of $A$. schaalii infections, which 
has led to suggestions of prolonged treatment $[6,19]$. The median length of antibiotic treatment in this series was 2 weeks and, to our knowledge, there were no treatment failures. One patient had two episodes of $A$. schaalii bacteremia during a 6month period, and this represented a recurrent infection rather than a treatment failure.

In conclusion, our results demonstrate that Actinotignum bacteremia is more common than previously thought and that it represents a condition with a significant fatality in elderly patients with underlying conditions. The urinary tract should be suspected as the primary focus of infection but, unfortunately, Actinotignum species seldom grow in ordinary urine culture conditions.

Acknowledgements We acknowledge the help of Dr. Bo Nilson, Dr. Karin Holm, and Mrs. Gisela Hovold.

\section{Compliance with ethical standards}

Funding This work was supported by the Swedish Government Fund for Clinical Research (ALF), the Royal Physiographic Society in Lund, and the foundations of Marianne and Marcus Wallenberg, Crafoord, Österlund, Tornspiran, and the Skåne University Hospital.

Conflict of interest The authors declare that they have no conflict of interest.

Ethical approval All procedures performed in studies involving human participants were in accordance with the ethical standards of the institutional and/or national research committee and with the 1964 Helsinki declaration and its later amendments or comparable ethical standards. The local ethics committee approved this study (reference number 2013/13).

Informed consent For this type of study, formal consent is not required.

Open Access This article is distributed under the terms of the Creative Commons Attribution 4.0 International License (http:// creativecommons.org/licenses/by/4.0/), which permits unrestricted use, distribution, and reproduction in any medium, provided you give appropriate credit to the original author(s) and the source, provide a link to the Creative Commons license, and indicate if changes were made.

\section{References}

1. Lawson PA, Falsen E, Akervall E, Vandamme P, Collins MD (1997) Characterization of some Actinomyces-like isolates from human clinical specimens: reclassification of Actinomyces suis (Soltys and Spratling) as Actinobaculum suis comb. nov. and description of Actinobaculum schaalii sp. nov. Int J Syst Bacteriol 47(3):899-903

2. Yassin AF, Spröer C, Pukall R, Sylvester M, Siering C, Schumann P (2015) Dissection of the genus Actinobaculum: Reclassification of Actinobaculum schaalii Lawson et al. 1997 and Actinobaculum urinale Hall et al. 2003 as Actinotignum schaalii gen. nov., comb. nov. and Actinotignum urinale comb. nov., description of
Actinotignum sanguinis sp. nov. and emended descriptions of the genus Actinobaculum and Actinobaculum suis; and re-examination of the culture deposited as Actinobaculum massiliense CCUG 47753T (= DSM 19118T), revealing that it does not represent a strain of this species. Int J Syst Evol Microbiol 65(Pt 2):615-624

3. Tuuminen T, Suomala P, Harju I (2014) Actinobaculum schaalii: identification with MALDI-TOF. New Microbes New Infect 2(2): $38-41$

4. Pearce MM, Hilt EE, Rosenfeld AB, Zilliox MJ, Thomas-White K, Fok $C$ et al (2014) The female urinary microbiome: a comparison of women with and without urgency urinary incontinence. MBio 5(4), e01283-14

5. Lotte R, Lotte L, Ruimy R (2016) Actinotignum schaalii (formerly Actinobaculum schaalii): a newly recognized pathogen-review of the literature. Clin Microbiol Infect 22(1):28-36

6. Nielsen HL, Søby KM, Christensen JJ, Prag J (2010) Actinobaculum schaalii: a common cause of urinary tract infection in the elderly population. Bacteriological and clinical characteristics. Scand J Infect Dis 42(1):43-47

7. Bank S, Jensen A, Hansen TM, Søby KM, Prag J (2010) Actinobaculum schaalii, a common uropathogen in elderly patients, Denmark. Emerg Infect Dis 16(1):76-80

8. Bank S, Søby KM, Kristensen LH, Voldstedlund M, Prag J (2015) A validation of the Danish microbiology database (MiBa) and incidence rate of Actinotignum schaalii (Actinobaculum schaalii) bacteraemia in Denmark. Clin Microbiol Infect 21(12):1097.e1-1097.e4

9. Hoenigl M, Leitner E, Valentin T, Zarfel G, Salzer HJ, Krause R et al (2010) Endocarditis caused by Actinobaculum schaalii, Austria. Emerg Infect Dis 16(7):1171-1173

10. Vanden Bempt I, Van Trappen S, Cleenwerck I, De Vos P, Camps K, Celens A et al (2011) Actinobaculum schaalii causing Fournier's gangrene. J Clin Microbiol 49(6):2369-2371

11. Lotte R, Durand M, Mbeutcha A, Ambrosetti D, Pulcini C, Degand $\mathrm{N}$ et al (2014) A rare case of histopathological bladder necrosis associated with Actinobaculum schaalii: the incremental value of an accurate microbiological diagnosis using $16 \mathrm{~S}$ rDNA sequencing. Anaerobe 26:46-48

12. Cattoir V (2012) Actinobaculum schaalii: review of an emerging uropathogen. J Infect 64(3):260-267

13. Sandlund J, Glimåker M, Svahn A, Brauner A (2014) Bacteraemia caused by Actinobaculum schaalii: an overlooked pathogen? Scand J Infect Dis 46(8):605-608

14. Gomez E, Gustafson DR, Rosenblatt JE, Patel R (2011) Actinobaculum bacteremia: a report of 12 cases. J Clin Microbiol 49(12):4311-4313

15. Non LR, Nazinitsky A, Gonzalez MD, Burnham CA, Patel R (2015) Actinobaculum schaalii bacteremia: a report of two cases. Anaerobe 34:84-85

16. Beguelin C, Genne D, Varca A, Tritten ML, Siegrist HH, Jaton K et al (2011) Actinobaculum schaalii: clinical observation of 20 cases. Clin Microbiol Infect 17(7):1027-1031

17. Hesstvedt L, Hasseltvedt V, Aandahl E, Caugant D, Høiby EA (2006) Septicaemia due to Actinobaculum schaalii. Scand J Infect Dis 38(8):735-737

18. Sturm PD, Van Eijk J, Veltman S, Meuleman E, Schülin T (2006) Urosepsis with Actinobaculum schaalii and Aerococcus urinae. J Clin Microbiol 44(2):652-654

19. Reinhard M, Prag J, Kemp M, Andresen K, Klemmensen B, Højlyng N et al (2005) Ten cases of Actinobaculum schaalii infection: clinical relevance, bacterial identification, and antibiotic susceptibility. J Clin Microbiol 43(10):5305-5308

20. Lotte L, Lotte R, Durand M, Degand N, Ambrosetti D, Michiels JF et al (2016) Infections related to Actinotignum schaalii (formerly Actinobaculum schaalii): a 3-year prospective observational study on 50 cases. Clin Microbiol Infect 22(4):388-390 
21. Cattoir V, Varca A, Greub G, Prod'hom G, Legrand P, Lienhard R (2010) In vitro susceptibility of Actinobaculum schaalii to 12 antimicrobial agents and molecular analysis of fluoroquinolone resistance. J Antimicrob Chemother 65(12):2514-2517

22. Statistiska centralbyrån (SCB) (2016) Folkmängden efter region civilstånd, ålder och kön. År 1968-2015, updated 160222. Statistiska centralbyrån, Statistics Sweden. Available online at: http://www.statistikdatabasen.scb.se/pxweb/sv/ssd/START BE BE0101_BE0101A/BefolkningNy/table/tableViewLayout1 /?rxid=634437cd-4d16-48f5-8168-17355fb9249c.

23. Senneby E, Göransson L, Weiber S, Rasmussen M (2016) A population-based study of aerococcal bacteraemia in the MALDITOF MS-era. Eur J Clin Microbiol Infect Dis 35(5):755-762

24. Kahn F, Linder A, Petersson AC, Christensson B, Rasmussen M (2010) Axillary abscess complicated by venous thrombosis: identification of Streptococcus pyogenes by 16S PCR. J Clin Microbiol 48(9):3435-3437

25. Brink M, Cronqvist J, Furebring M, Gille-Johnson P, Gårdlund B, Lanbeck P et al (2013) Svår sepsis och septisk chock - tidig identifiering och initial handläggning, updated December 2013. Programgrupp Sepsis. Available online at: http://www.infektion.
net/sites/default/files/Sv\%C3\%A5r_sepsis_och_septisk_chock dec 2013 new.pdf.

26. European Committee on Antimicrobial Susceptibility Testing (EUCAST) (2016) Breakpoint tables for interpretation of MICs and zone diameters. Version 6.0. Available online at: http://www. eucast.org/clinical_breakpoints/.

27. Rasmussen M (2016) Aerococcus: an increasingly acknowledged human pathogen. Clin Microbiol Infect 22(1):22-27

28. Senneby E, Eriksson B, Fagerholm E, Rasmussen M (2014) Bacteremia with Aerococcus sanguinicola: case series with characterization of virulence properties. Open Forum Infect Dis 1(1), ofu025

29. Senneby E, Petersson AC, Rasmussen M (2012) Clinical and microbiological features of bacteraemia with Aerococcus urinae. Clin Microbiol Infect 18(6):546-550

30. Christensen JJ, Jensen IP, Faerk J, Kristensen B, Skov R, Korner B (1995) Bacteremia/septicemia due to Aerococcus-like organisms: report of seventeen cases. Danish ALO Study Group. Clin Infect Dis 21(4):943-947

31. Sunnerhagen T, Nilson B, Olaison L, Rasmussen M (2016) Clinical and microbiological features of infective endocarditis caused by aerococci. Infection 44(2):167-173 